\title{
Hydrodynamic models of gas-liquid two-phase flow in porous media
}

\author{
Blanca Gutiérrez R. ${ }^{1, *}$, Rafael Álvarez B. ${ }^{2}$ \\ and Aura L. López de Ramos ${ }^{3}$ \\ GFT $_{\text {usB }}$, Universidad Simón Bolívar, Apartado Postal 89000. Caracas, \\ Venezuela. \\ 1'bgutierrez@usb.ve, ${ }^{2}$ rafalvar@usb.ve, ${ }^{3}$ alopez@usb.ve
}

\begin{abstract}
Equations and models describing the hydrodynamic of gas-liquid twophase flows in porous media have become increasingly necessary in order to predict their main features throughout porous networks. The main subject of this research was to study the influence of capillary, viscous and inertial forces and flow configurations on the hydrodynamic features of a gas-liquid two-phase flow in a glass micromodel. Experimental results were obtained and compared with those predicted by three published models. The Fundamental Forces Balance and the Fluid-Fluid Interface models did not describe accurately experimental behavior even when the first of them considers particular characteristics of flow patterns. Semi-empirical models such as The Relative Permeability can describe physical flow characteristics and can also be modified to include different effects not initially considered. Traditionally, relative permeabilities have been associated almost exclusively with saturation conditions. However, it was concluded in this research that liquid relative permeability is function of saturation conditions but also depends on flow patterns and Capillary number.
\end{abstract}

Key words: hydrodynamic models; gas-liquid two-phase flow; flow patterns; relative permeabilities; porous media

\section{NOMENCLATURE}

$A_{T} \quad$ transverse area of the porous medium $\left[\mathrm{m}^{2}\right]$

Bo Bond number, $\left(B o=\left(\rho_{L}-\rho_{G}\right) \cdot g \cdot L_{C}^{2} / \sigma\right)$, [dimensionless]

$C a_{\mathrm{i}} \quad$ capillary number, $\left(C a_{i}=\mu_{i} \cdot u_{i} / \sigma\right)$, [dimensionless]

$d_{\mathrm{i}} \quad$ diameter $[\mathrm{m}]$

$f_{G} \quad$ geometric factor [dimensionless]

$f_{\text {Wall }} \quad$ walls shear stress on liquid phase $\left[\mathrm{N} / \mathrm{m}^{3}\right]$

$F_{\mathrm{i}} \quad$ drag force $[\mathrm{N}]$

$F_{\mathrm{ij}} \quad$ interaction forces of phases $i$ and $j$ per unit of total volume $\left[\mathrm{N} / \mathrm{m}^{3}\right]$

$g \quad$ gravity acceleration $\left[\mathrm{m} / \mathrm{s}^{2}\right]$

$G a_{\mathrm{i}}{ }^{*} \quad$ Galileo number modified, $\left(G a_{i}^{*}=\rho_{i}^{2} \cdot g \cdot d_{P}^{3} \cdot \varphi^{3} /\left(\mu_{i}^{2} \cdot(1-\varphi)^{3}\right)\right)$, [dimensionless]

$k \quad$ absolute permeability $\left[\mathrm{m}^{2}\right]$

$k_{r \mathrm{i}} \quad$ relative permeability $\left[\mathrm{m}^{2}\right]$

$l_{\mathrm{i}} \quad$ length $[\mathrm{m}]$

$L_{C} \quad$ characteristic length $[\mathrm{m}]$

*Corresponding author. E-mail: bgutierrez@usb.ve 
$N \quad$ data number [dimensionless]

$N_{B} \quad$ bubbles number [dimensionless]

$p_{\mathrm{i}} \quad$ pressure $[\mathrm{Pa}]$

$Q_{i} \quad$ volumetric flow $\left[\mathrm{m}^{3} / \mathrm{s}\right]$

$R e_{\mathrm{i}} \quad$ Reynolds number, $\left(\operatorname{Re}=\rho_{i} \cdot u_{i} \cdot L_{C} / \mu_{i}\right)$, [dimensionless]

$\operatorname{Re}_{\mathrm{i}}^{*} \quad$ Reynolds number modified, $\left(\operatorname{Re}_{i}=\rho_{i} \cdot u_{i} \cdot d_{P} / \mu_{i} \cdot(1-\varphi)\right)$, [dimensionless]

$S_{\mathrm{i}} \quad$ saturation [dimensionless]

$S_{i r} \quad$ reduced saturation of the phase [dimensionless]

$S_{i O} \quad$ irreducible saturation [dimensionless]

$u_{\mathrm{i}} \quad$ superficial Darcy's velocity $[\mathrm{m} / \mathrm{s}]$

$u_{D} \quad$ relative velocity of bubbles respect to the gas-liquid flow $[\mathrm{m} / \mathrm{s}]$

$X_{C} \quad$ predicted value of variable $X$

$X_{E} \quad$ experimental value of variable $X$

$z \quad$ elevation $[\mathrm{m}]$

$\left\langle e_{X}\right\rangle \quad$ average relative error of variable $X$ [dimensionless]

\section{GREEK SYMBOLS}

$\phi \quad$ average porosity of the porous medium [dimensionless]

$\mu_{\mathrm{i}} \quad$ dynamic viscosity [Pa.s]

$\rho_{\mathrm{i}} \quad$ density $\left[\mathrm{kg} / \mathrm{m}^{3}\right]$

$\sigma \quad$ surface tension $[\mathrm{N} / \mathrm{m}]$

$\begin{array}{ll}\text { SUBSCRIPTS } \\ \mathrm{B} & \text { bubble } \\ \text { W } & \text { wetting phase } \\ \text { NW } & \text { non wetting phase } \\ \text { G } & \text { gas phase } \\ \text { L } & \text { liquid phase } \\ \text { P } & \text { particle }\end{array}$

\section{INTRODUCTION}

Equations and models describing hydrodynamics of gas-liquid two-phase flows in porous media are particularly important in areas such as the treatment of contaminated soils, enhanced recovery processes, nuclear reactors, multiphase reactors, packed beds, and others. Since late $19^{\text {th }}$ century some empirical models have been used in reservoir engineering but they have failed reproducing the actual flows velocities obtained during exploitation. Therefore, new models are required to represent the main hydrodynamic features in order to predict accurately the fluids flows rate during the reservoir exploitation. Some of the models already developed are the force balance model [1], the fluid-fluid interfacial model [2] and the relative permeability model [3]. Drag forces of each of the phases are considered on two of those models, the force balance model [1] and the fluid-fluid interfacial model [2], which are based on momentum balance in the fluid-fluid and fluid-solid interfaces at pore scale. These models do not have parameters that could be adjusted or modified to account any other effect on the transport phenomenon [4].

The relative permeability model was developed on macroscopic scale and it was obtained from Ergun's equation [3]. Viscous and inertial forces in the transport of single-phase flow in packed beds and the absolute permeability of the medium were considered in this model. Since Darcy's law was generalized to multiphase flow through porous media, relative 
permeabilities have been associated just with the saturation of the phases. Furthermore, separated and connected flow of each one of the phases is the main assumption in the generalized Darcy's law. The relevance of the actual viscous effects has been discussed by many authors [5]. It has been observed that generalized Darcy's law is inaccurate when fluids flow disconnected in the porous media [5],[6].

Relative permeability concept has been used in many and different porous systems such trickled bed reactors and bubble columns. However, Sáez \& Carbonell [3] developed for the first time a model where relative permeabilities are the main correction factors introduced in order to quantify the simultaneously and disconnected flow of two immiscible phases in a porous media. Empirical correlations are used in this model to calculate the relative permeabilities of the phases. Despite this, some authors such Nemec et al. [7] and Atta et al. [8] conclude that the relative permeability model is a simple and accurate manner to characterize hydrodynamic two-phase flow in porous media. Also numerical simulations based on this model and using CFD techniques [8] have been developed. The results obtained have been agreed with experimental observations in porous networks.

Several studies [9],[10],[11] have been made in order to quantify the dependence of relative permeability $\left(k_{r \mathrm{i}}\right)$ with Reynolds number $\left(R e_{\mathrm{i}}\right)$, viscosity of the phases $\left(\mu_{\mathrm{i}}\right)$, surface tension $(\sigma)$, superficial velocities of the phases $\left(u_{\mathrm{i}}\right)$, and others. Those studies concluded that relative permeability only depends on saturation of the phases. Some authors [12],[13],[14],[15],[16] claim that viscosity ratio, capillary number, flow configurations and others parameters should be properly considered in hydrodynamic studies of two-phase flow in porous media. Most of that research work has been developed on liquid-liquid systems.

Sáez et al. [11] developed theoretical models in order to calculate relative permeabilities using straight and tortuous capillaries. These authors using the finite elements method (FEM) to solve momentum equations concluded that $k_{r \mathrm{G}}$ was a function of $R e_{\mathrm{G}}$, especially when $R e_{\mathrm{G}}$ was low. This dependence disappeared when $R e_{\mathrm{G}}$ was increased. They did not take into account flow configurations on $k_{r i}$.

Complex structure of the porous medium and interfacial interactions between the fluids are the major obstacles to overcome in hydrodynamic studies of two-phase flow in order to obtain purely theoretical models. Therefore, adjustment of certain parameters is necessary [7],[8]. Indeed, empirical and semi-empirical models are frequently used to represent the hydrodynamic of these systems [17]. The main advantage of those empirical and semiempirical models is the presence of adjustable parameters, which could be adjusted to include the influence of flow configurations, viscous effects, interfacial interactions, and any other phenomenon not considered into theoretical models.

The main subject of this research was to evaluate three published hydrodynamic models and comparing these predicted values with experimental results obtained in a $2 \mathrm{D}$ glass micromodel when a gas-liquid two-phase flow were used. Moreover, it was studied the influence of capillary number and flow configurations in the hydrodynamic features.

\section{EXPERIMENTAL SETUP}

The experimental setup showed in Figure 1 was designed to determine steady-state gasliquid two-phase flow variables and is fully explained in a previous experimental work [18]. Air coming from a gas cylinder (AC) was used as the gas phase. The liquid stored in a tank (ST) was impelled by a positive displacement pump (P) and regulated with a needle valve (V1). The gas-liquid two-phase flow entered simultaneously to the porous glass micromodel (GM) and the pressure transducer (T). An indicator (I) was used to register the pressure drop inside the micromodel. 


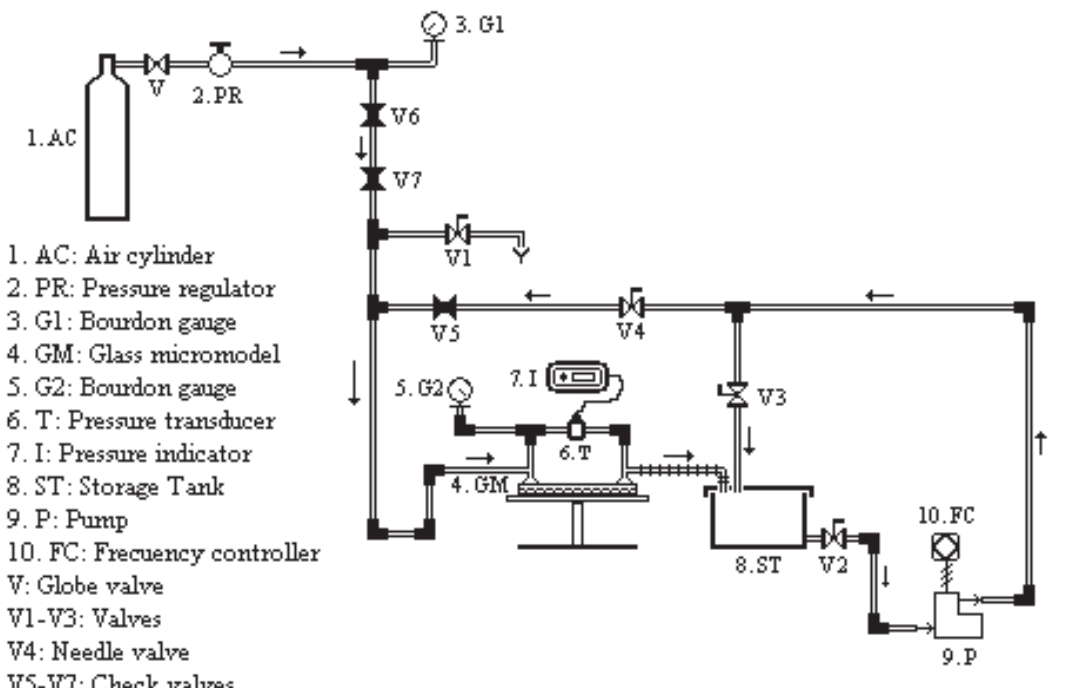

Figure 1. Experimental setup.

The porous glass micromodel used in this study has $53 \%$ porosity $(\phi)$, an absolute permeability $(k)$ of 18 Darcy, a transverse area $\left(A_{T}\right)$ of $9 \mathrm{~mm}^{2}$, and $0.1 \mathrm{~mm}$ of width. It can be observed in Figure 2 some of the characteristics of the porous glass micromodel.

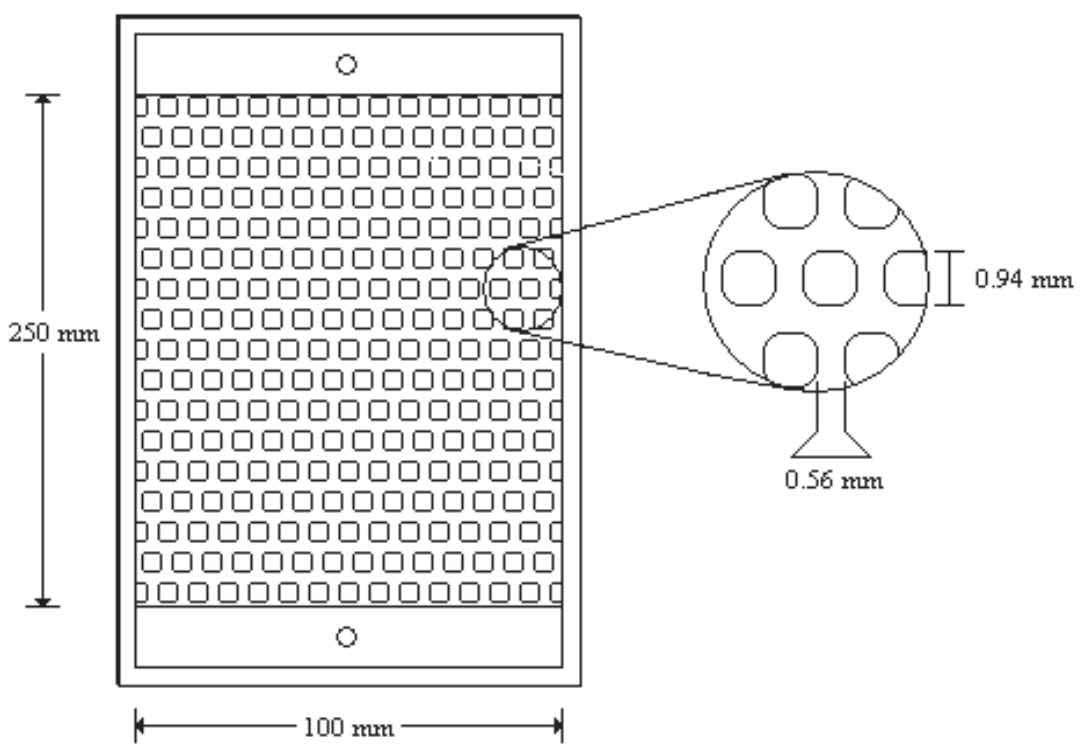

Figure 2. Glass micromodel. 
Diluted solutions of glycerin were used as the liquid phase. Surface tension was modified with a non-ionic surfactant. Physical properties of the liquid phase are shown in Table 1.

Table 1. Physical properties of the liquid phase.

\begin{tabular}{|c|c|c|c|}
\hline Substance & $\begin{array}{c}\text { Density } \\
(\rho \pm 1) \mathrm{kg} / \mathrm{m}^{3}\end{array}$ & $\begin{array}{l}\text { Viscosity } \\
\mu \text { mPa.s }\end{array}$ & $\begin{array}{l}\text { Surface tension } \\
(\sigma \pm 0,1) \mathrm{mN} / \mathrm{m}\end{array}$ \\
\hline Water & 1048 & \multirow{2}{*}{$1,0 \pm 0,1$} & 72,5 \\
\hline Water-Tritón ${ }^{\mathrm{TM}} \mathrm{X}-100$ & 1043 & & $53,1^{*}$ \\
\hline AG-1 & 1139 & \multirow{2}{*}{$10 \pm 1$} & 68,1 \\
\hline AGT-1 & 1149 & & $50,7^{*}$ \\
\hline $\mathrm{AG}-2$ & 1168 & \multirow{2}{*}{$20 \pm 1$} & 66,4 \\
\hline AGT-2 & 1166 & & $50,4^{*}$ \\
\hline AG-3 & 1185 & \multirow{2}{*}{$30 \pm 1$} & 66,0 \\
\hline AGT-3 & 1198 & & $50,3^{*}$ \\
\hline AG-4 & 1202 & \multirow{2}{*}{$40 \pm 1$} & 65,7 \\
\hline AGT-4 & 1205 & & $50,2^{*}$ \\
\hline
\end{tabular}

*These values correspond to the critic micelle concentration of Tritón TM X-100.

In this study, the visualization system consisted of a high-speed camera, lens, a monitor, and a workstation. This equipment was used to capture and record the gas-liquid two-phase flow in the porous glass micromodel. The high-speed video camera was mounted over the glass micromodel to capture the two-phase flow on the central zone of the porous medium. Additionally, a precise scale was recorded and used later to calibrate the image pixel resolution.

\subsection{METHODOLOGY}

Once the glass micromodel was saturated with the wetting phase, the non-wetting phase was injected and the pressure for each test was adjusted to a prefixed value. In this way, the wetting phase was displaced by the non-wetting phase, which describes a drainage process. This was done increasing progressively the $Q \mathrm{NW} / Q \mathrm{~W}$ ratio. The steady-state condition was obtained as soon as the pressure drop in the micromodel remained constant, which were registered as experimental results. At that moment, images of the flow in the glass micromodel were recorded in order to calculate the saturation of both of the phases.

Once the steady-state flow conditions were established and the boundary effect neglected [18], it was possible to assume that the capillary pressure in the micromodel was uniform, and hence,

$$
\Delta p_{\mathrm{W}}=\Delta p_{\mathrm{NW}}
$$

In all experimental tests the liquid volumetric flow $\left(Q_{\mathrm{L}}\right)$ and the gas superficial velocity $\left(u_{\mathrm{G}}\right)$ ranged from 0.3 to $3.0 \mathrm{ml} / \mathrm{min}$ and from 18 to $27 \mathrm{~m} / \mathrm{s}$, respectively. Capillary number 
$\left(C a_{L}\right)$ and Reynolds number $\left(R e_{L}\right)$ ranged from $3 \times 10^{-4}$ to $1.5 \times 10^{-3}$ and from $9.8 \times 10^{-5}$ to $3.0 \times 10^{-3}$, respectively. Gravitational effects were neglected since the Bond's number $(B o)$ calculated from experimental values varied from $5.9 \times 10^{-7}$ to $1.2 \times 10^{-5}$.

\section{RESULTS}

The subsequent analysis is exclusively focused on the continuous liquid phase.

Two different flow patterns were observed in the glass micromodel, bubble flow and slug flow. Image processing techniques (IPT) were used to characterize both configurations. Detailed information about these techniques and the characterization of the flow patterns observed in the glass micromodel would be available in Gutierrez et al. [19]. While the bubble flow was characterized by a large number of small spherical bubbles, the slug flow was characterized by fewer elongated bubbles. Diameter and length of the bubbles were some of the results obtained with the IPT developed [19]. The average ratio $l_{B} / d_{B}$ calculated was 0.62 and 1.92 for bubble and slug flow, respectively.

Three hydrodynamic models of two-phase flow were selected to compare with those results obtained experimentally in the glass micromodel: (1) fundamental forces balance or FFBM [1], (2) fluid-fluid interface or FFIM [2] and (3) relative permeability or RPM [3]. A disconnected gas flow in a continuous liquid phase is the common assumption in all these models. This was observed in both flow patterns obtained in the glass micromodel.

Equations of every model evaluated can be consulted in Appendix A. Deviation between experimental and theoretical values were quantified through average relative errors $\left(\left\langle e_{X^{\prime}}\right)\right.$ :

$$
\left\langle e_{X}\right\rangle=\frac{1}{N} \cdot \sum_{i=1}^{N} \frac{\left|X_{C}-X_{E}\right|}{X_{E}} \cdot 100
$$

\subsection{FUNDAMENTAL FORCES BALANCE MODEL (FFBM)}

Theoretical values of pressure drop obtained from Eqns. (A-1) to (A-10) corresponding to the FFBM [1] and the experimental values can be observed in Figure 3. The average relative error was $51 \%$ and $87 \%$ for slug flow and bubble flow, respectively. This considerable

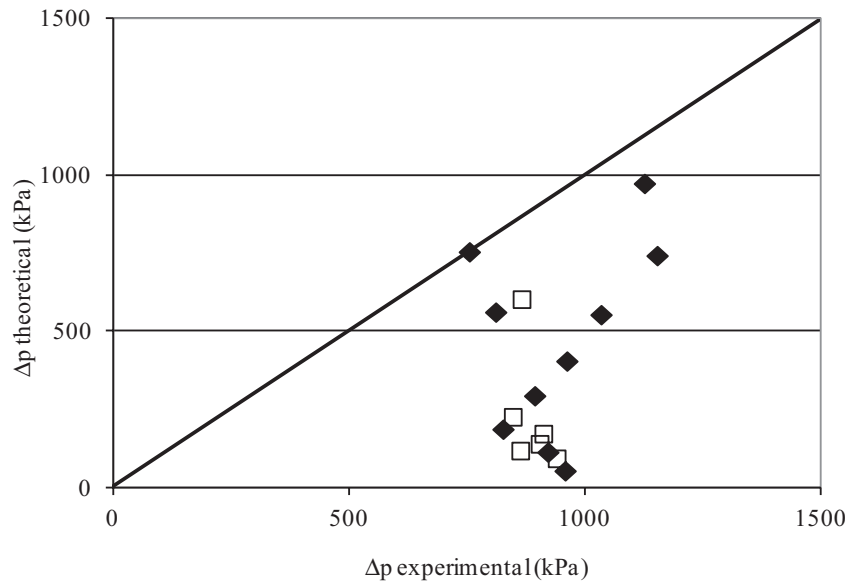

Figure 3. Pressure drop; experimental: $\diamond$ slug flow, $\square$ bubble flow; theoretical FFBM [1]. 
deviation is consequence of the $800 \%$ of $\left\langle e_{X}\right\rangle$ obtained when the theoretical diameter, Eqn. (A-8), were compared with the actual averaged values obtained from IPT [19]. This deviation is propagated when the average bubbles number was calculated with Eqn. (A.9), which was considerably above the actual experimental value. Coalescence and rupture of the bubbles were not considered in the model proposed by Tung \& Dhir [1]. Both phenomena were observed in both flow configurations especially in bubble flows. This could explain the higher value of $\left\langle e_{X}\right\rangle$ obtained.

\subsection{FLUID-FLUID INTERFACE MODEL (FFIM)}

Theoretical values of pressure drop obtained from Eqns. (A-11) to (A-15) corresponding to the FFIM [2] and the experimental ones are presented in Figure 4. The average relative error obtained were $56 \%$ and $62 \%$ for slug and bubble flow, respectively. This model also overestimate the bubbles diameter with $\left\langle e_{X^{\prime}}=171 \%\right.$ calculated from Eqn. (A-14) and consequently the bubbles number, Eqn. (A-15). Attou \& Ferschneider [2] also ignored breakup and coalescence of gas bubbles in the porous media. The exact same size for all the bubbles in the liquid flow is one of the main assumptions in both FFBM and FFBM, which was not agreed with the observations made in the glass micromodel. Coalescence and breakup of bubbles caused a gas flow polidisperse. Collisions between gas bubbles were frequently observed in the flow images, especially in bubble flows no matter $C a_{\mathrm{L}}$ or $R e_{\mathrm{G}}$ [19]. Coalescence, breakup, and collisions were not considered by these authors when they developed the model.

All the phenomena previously described and not considered in FFBM and FFIM have a significant influence in drag forces of the gas phase and hence the pressure drop in the glass micromodel. Also, it was observed a large number of stagnate bubbles, which is another phenomenon not considered in these models and that seems to have a considerable relevance in hydrodynamic features in a porous media.

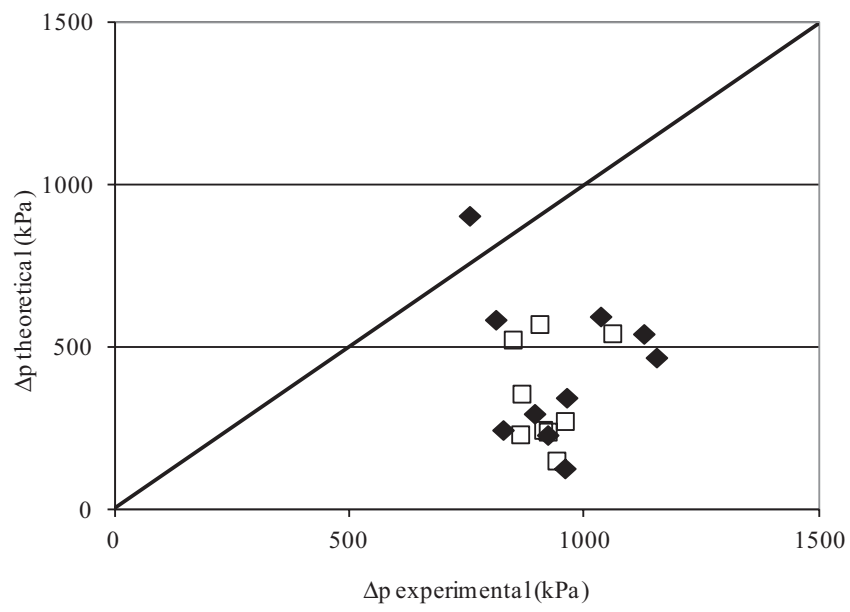

Figure 4. Pressure drop; experimental: $\diamond$ slug flow, $\square$ bubble flow; theoretical FFIM [2]. 


\subsection{RELATIVE PERMEABILITY MODEL (RPM)}

In general, this model is frequently used due its simplicity and accuracy in modeling twophase flows in porous media [4]. In this research Eqns. (A-16) and (A-17) were used to calculate relative permeability of the liquid phase using experimental pressure drop, density and viscosity of the liquid phase. The models selected to predict $k_{r L}$ were those proposed by Sáez \& Carbonell [3], Eqn. (A-18), Lakota et al. [20], Eqn. (A-19), and Corey [21], Eqn. (A20). This last equation is the most common one used in reservoir engineering including commercial software developed to simulate and estimate different production flow rates in oil wells under several circumstances.

Relative permeabilities curves of the liquid phase obtained from selected and cited models, Eqns. (A-18), (A-19), and (A-20) are shown in Figure 5.

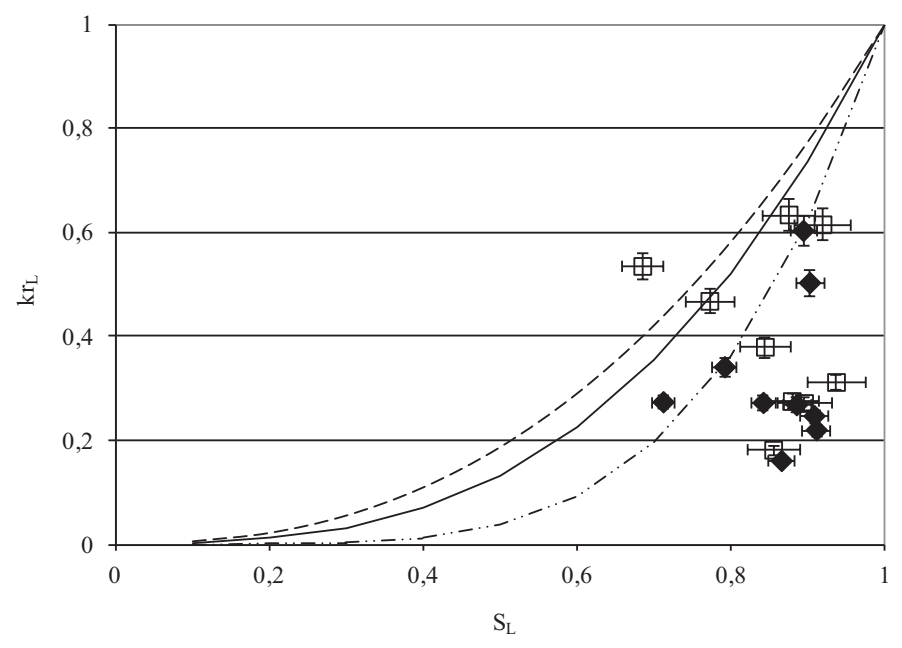

Figure 5. Relative permeability curves of liquid phase: $\downarrow$ slug flow; $\square$ bubble flow; - - Sáez \& Carbonell [3]; - Lakota et al. [20]; -..- Corey [21].

In Figure 5 can be observed that neither of the correlations used and proposed by the cited authors predicted accurately the corresponding experimental values in the glass micromodel obtained in this research. Such discrepancy is consequence of the assumed theoretical condition that $k_{r \mathrm{~L}}$ is an exclusive function of its own saturation, which is very common in most of the hydrodynamic studies of two-phase flow [3],[9],[17]. It can be seen clearly in Figure 5 that one or more factors besides the liquid saturation have some influence on relative permeabilities of the connected phase when a gas-liquid two-phase flow circulates in a glass micromodel. This might explain the average relative errors presented in Table 2. 
Table 2. Average relative errors of the evaluated models of $k_{r L}$ respect to the correspondent experimental values.

\begin{tabular}{cc}
\hline Model & $\left\langle\boldsymbol{e}_{X^{\rangle}}(\mathbf{\%})\right.$ \\
\hline Sáez \& Carbonell [3] & 113 \\
\hline Lakota et al. $[20]$ & 101 \\
\hline Corey [21] & 80 \\
\hline
\end{tabular}

Several authors [9],[10],[17] have been concluded from their own research work a completely independence of $k_{r \mathrm{~L}}$ from physical properties such the density and the viscosities of the phases and the geometric pattern of the medium.

Regarding all the previous discussion and considering a completely absence of a clear relationship of the $k_{r \mathrm{~L}}$ just with $S_{\mathrm{L}}$; in this research is presumed a direct influence of capillary and inertial forces and flow configurations in the relative permeabilities curves of the liquid phase, i.e. in the overall hydrodynamic features of two-phase flow in porous media. Avraam \& Payatakes [14] reported an influence of the flow configurations in the hydrodynamic of liquid-liquid two-phase flow. Ostos \& Maini [15] reported that gas-liquid relative permeabilities are related with $\mathrm{Ca}$. However, they did not propose any correlation between the factors studied. A very few work research has been done using gas-liquid two-phase flow when one of the phases flows disconnected.

\section{CONCLUSIONS}

Hydrodynamic models purely theoretical such the fundamental forces balance FFBM [1] and the fluid-fluid interfacial model FFIM [2] do not describe the main hydrodynamic features of the gas-liquid two-phase flow in the glass micromodel measured experimentally in this research. Despite Tung \& Dhir [1] considered flow patterns in their model; the predicted values do not match with those experimental values obtained. Instead, semi-empirical models, such RPM [3] based on solid physical principles could be modified through adjustable parameters to introduce the dependence on flow patterns and some other effect observed experimentally and not initially considered in the model. This is the main advantage of these semi-empirical models.

Relative permeabilities have been associated almost exclusively just with phase saturation [3],[9],[17]. However, in this research it was concluded that $k_{r \mathrm{~L}}$ is not just function of $S_{L}$ but also depends on variables such flow patterns, capillary forces, and interactions between bubbles. Our future research work will be orientated to obtain correlations to describe in a more accurate way the main hydrodynamic features of gas-liquid two-phase flow in a 2D porous medium, which could be a major advance in the comprehension of these phenomena at pore and reservoir scale.

\section{REFERENCES}

[1] Tung, V. X., \& Dhir V. K. A hydrodynamic model for two-phase flow through porous media, Int. J. Multiphase Flow, 1988, 14(1), 47-65.

[2] Attou, A., \& Ferschneider, C. A simple model for pressure drop and liquid hold-up in packed-bed bubble reactors. Chem. Eng. Sci., 1999, 54, 5139-5144. 
[3] Sáez, A. E., \& Carbonell, R. G. Hydrodynamics Parameters for Gas-Liquid Cocurrent Flow in Packed Beds. AIChE Journal, 1985, 31(1), 52-62.

[4] Carbonell, R. G. Multiphase Flow Models in Packed Beds. Oil Gas Sci. Technol., 2000, 55, 4, 417-425.

[5] Bravo, M., Araujo, M., \& Lago, M. Pore network modeling of two-phase flow in a liquid(disconnected) gas system. Physica A, 2007, 375, 1-17.

[6] Kamp, A., Heny, C., Andarcia, L., Lago, M., \& Rodriguez, A. Experimental Investigation of Foamy Oil Solution Gas Drive. SPE 69725, 2001, presented at the SPE International Thermal Operations and Heavy Oil Symposium, Porlamar, Venezuela.

[7] Nemec, D., Bercic, G., \& Levec, J. The hydrodynamics of trickling flow in packed beds operating at high pressures. The relative permeability concept. Chem. Eng. Sci., 2001, 56, 5955-5962.

[8] Atta, A., Roy, S., \& y Nigam, K. D. P. Prediction of pressure drop and liquid holdup in trickle bed reactor using relative permeability concept in CFD. Chem. Eng. Sci., 2007, 62, 5870-5879.

[9] Osoba, J. S., Richardson, J. G, Kerver, J. K, Hafford J. A \& Blair P. M. Laboratory Measurements of Relative Permeability. Trans. AIME, 1951, 192, 47-56.

[10] Richardson, J. G., Kerver, J. K., Hafford, J. A., \& Osoba, J. S. Laboratory Determination of Relative Permeability. Trans. AIME, 1952, 195, 187-197.

[11] Sáez, A. E., Carbonell, R. G. \& Levec, J. The Hydrodynamics of Trickling Flow in Packed Beds. AIChE Journal, 1986, 32(3), 353-368.

[12] Fulcher, R. A., Ertekin, T., \& Stahi, C. D. The Effect of the Capillary Number and Its Constituents on Two-Phase Relative Permeability Curves. SPE 12170, 1983, presented at the SPE Annual Technical Conference and Exhibition, San Francisco, California.

[13] Avraam D., Kolonis, G., Roumeliotis, T., Constantinides, G., \& Payatakes, A. Steady-State TwoPhase Flow Through Planar and Nonplanar Model Porous Media. Transp. Porous Media, 1994, $16,75-101$.

[14] Avraam, D., \& Payatakes, A. Generalized Relative Permeabilities Coefficients during SteadyState Two-Phase Flow in Porous Media, and Correlation with Flow Mechanisms. Transp. Porous Media, 1995, 20, 135-168.

[15] Ostos, A., \& Maini, B. Capillary Number in Heavy Oils Solution Gas Drive and Its Relationship with Gas-Oil Relative Permeability Curve. SPE 89430, 2004, presented at the SPE/DOE $14^{\text {th }}$ Improved Oil Recovery, Tulsa, Oklahoma.

[16] Tsakiroglou, C., Avraam D., \& Payatakes, A. Transient and steady-state relative permeabilities from two-phase flow experiments in planar pore networks. Adv. Water Res., 2007, 30, 1981-1992.

[17] Nemec, D., \& Levec, J. Flow through packed bed reactor: 2. Two-phase concurrent downflow. Chem. Eng. Sci., 2005, 60, 6958-6970.

[18] Gutierrez, B., Juarez, F., Ornelas, L., Zeppieri, S. \& López de Ramos, A. Experimental Study of Gas-Liquid Two-Phase Flow in Glass Micromodels. Int. J. Thermophys., 2008, 29(6), 2126-2135.

[19] Gutierrez, B., Pirard, J., Blacher, S., \& López, A. (Submitted). Image Analysis of the Gas Morphology in a Gas-Liquid Two-Phase Flow in a Porous Glass Micromodel.

[20] Lakota, A., Levec, J., \& Carbonell, R. G. Hydrodynamics of trickling flow in packed beds: Relative permeability concept. A. I. Ch. E. Journal, 2002, 48(4), 731-738.

[21] Corey, A. The interrelation between gas and oil relative permeabilities. Producers Monthly, 1954, 19(1), 38-41. 
APPENDIX A

FFBM equations [1]: These authors proposed the following equations to model the liquid phase in a gas-liquid two-phase flow:

$$
\begin{aligned}
& -\varphi \cdot S_{\mathrm{L}} \frac{\mathrm{d} p_{\mathrm{L}}}{\mathrm{d} z}=\rho_{\mathrm{L}} \cdot g \cdot \varphi \cdot S_{\mathrm{L}}+F_{\mathrm{LS}}-F_{\mathrm{GL}} \\
& F_{\mathrm{LS}}=\varphi \cdot\left(\frac{a \cdot \mu_{\mathrm{L}} \cdot u_{\mathrm{L}}}{\left(1-S_{\mathrm{G}}\right)^{3}}+\frac{b \cdot \rho_{\mathrm{L}} \cdot u_{\mathrm{L}}^{2}}{\left(1-S_{\mathrm{G}}\right)^{3}}\right)
\end{aligned}
$$

$a$ and $b$ are the Kozeny-Carman equations.

Drag forces $F_{\mathrm{GL}}$ are calculated according to the flow configurations:

Bubble and slug flow $\left(0 \leq S_{G} \leq S_{\mathrm{G} 3}\right)$ :

$$
\begin{aligned}
& F_{\mathrm{GL}}=C_{V} \cdot \frac{\mu_{\mathrm{L}} \cdot u_{D}}{d_{\mathrm{B}}^{2}}+C_{I} \cdot \frac{\left(1-S_{\mathrm{G}}+\rho_{\mathrm{G}} / \rho_{\mathrm{L}} \cdot S_{\mathrm{G}}\right) \cdot \rho_{\mathrm{L}} \cdot u_{D}^{2}}{\varphi \cdot d_{\mathrm{B}}} \\
& C_{V}=18 \cdot S_{\mathrm{G}} \cdot f_{G} \\
& 0 \leq S_{G} \leq S_{\mathrm{G} 0} \quad C_{I}=0.34 \cdot\left(1-S_{\mathrm{G}}\right)^{3} \cdot S_{\mathrm{G}} \cdot f_{G}^{2} \\
& S_{\mathrm{G} 0} \leq S_{G} \leq S_{\mathrm{G} 1} \quad C_{V}=18 \cdot\left(S_{\mathrm{G} 0} \cdot f_{G}+S_{\mathrm{G}}-S_{\mathrm{G} 0}\right) \\
& C_{I}=0.34 \cdot\left(1-S_{\mathrm{G}}\right)^{3} \cdot\left(S_{\mathrm{G} 0} \cdot f_{G}^{2}+S_{\mathrm{G}}-S_{\mathrm{G} 0}\right) \\
& \begin{array}{ll}
S_{\mathrm{G} 2} \leq S_{G} \leq S_{\mathrm{G} 3} \quad C_{V}=5.21 \cdot S_{\mathrm{G}} \\
C_{I}=0.92 \cdot\left(1-S_{\mathrm{G}}\right)^{3} \cdot S_{\mathrm{G}}
\end{array} \\
& f_{G}=\frac{1}{2} \cdot\left(1+\frac{d_{\mathrm{B}}}{d_{\mathrm{P}}}\right) \ln \left(1+2 \cdot \frac{d_{\mathrm{B}}}{d_{\mathrm{P}}}\right) \\
& d_{\mathrm{B}}=1.35 \cdot\left(\frac{\sigma}{g \cdot\left(\rho_{\mathrm{L}}-\rho_{\mathrm{G}}\right)}\right)^{1 / 2} \\
& N_{B}=6 \cdot \frac{S_{\mathrm{G}} \cdot \varphi}{\pi \cdot d_{\mathrm{B}}^{3}}
\end{aligned}
$$




$$
u_{D}=u_{\mathrm{G}} \cdot \frac{\left(1-S_{\mathrm{G}}\right)}{S_{\mathrm{G}}}-u_{\mathrm{L}}
$$

FFIM equations [2]: These authors presented the following equations to represent the hydrodynamic of the liquid phase:

$$
\begin{aligned}
& S_{\mathrm{L}} \frac{\mathrm{d} p_{\mathrm{L}}}{\mathrm{d} z}=S_{\mathrm{L}} \cdot \rho_{\mathrm{L}} \cdot g+f_{\mathrm{GL}}-f_{\mathrm{LS}}-f_{\text {Wall }} \\
& f_{\mathrm{LS}}=\left(1-S_{\mathrm{G}}\right) \cdot\left(A_{L S} \cdot \mu_{\mathrm{L}} \cdot u_{\mathrm{L}}+B_{L S} \cdot \rho_{\mathrm{L}} \cdot u_{\mathrm{L}}^{2}\right)
\end{aligned}
$$

$A_{L S}$ and $B_{L S}$, are the Kozeny-Carman equations for pure liquid but corrected to account the presence of gas bubbles.

$$
f_{\mathrm{GL}}=S_{\mathrm{G}} \cdot\left(A_{G L} \cdot \mu_{\mathrm{L}} \cdot u_{D}+B_{G L} \cdot \rho_{m} \cdot u_{D}^{2}\right)
$$

$A_{G L}$ and $B_{G L}$ quantify the interaction forces originated mainly by viscous drag forces.

$$
\begin{aligned}
& d_{\mathrm{B}}=1,15 \cdot\left(\frac{\sigma}{\rho_{\mathrm{L}}}\right)^{3 / 5} \cdot\left(\frac{u_{\mathrm{L}}}{\varphi \cdot \rho_{\mathrm{L}}}\left(-\frac{\mathrm{d} p}{\mathrm{~d} z}+\rho_{\mathrm{L}} \cdot g\right)\right)^{-2 / 5} \\
& N_{B}=6 \cdot \frac{S_{\mathrm{G}}}{\pi \cdot d_{\mathrm{B}}^{3}}
\end{aligned}
$$

\section{RPM equations [3]:}

$$
\begin{aligned}
& \varphi \cdot S_{\mathrm{i}}\left(-\frac{\mathrm{d} p_{\mathrm{i}}}{\mathrm{d} z}+\rho_{\mathrm{i}} \cdot g\right)=F_{\mathrm{i}} \\
& \frac{F_{\mathrm{i}}}{\varphi \cdot S_{\mathrm{i}}}=\frac{1}{k_{r i}\left(S_{\mathrm{i}}\right)} \cdot\left(A \cdot \frac{\mathrm{Re}_{\mathrm{i}}^{*}}{G a_{\mathrm{i}}^{*}}+B \cdot \frac{\mathrm{Re}_{\mathrm{i}}^{2}}{G a_{\mathrm{i}}^{*}}\right) \cdot \rho_{\mathrm{i}} \cdot g
\end{aligned}
$$

Equations to determine relative permeabilities of liquid phase [3],[20],[21]:

$$
k_{r \mathrm{~L}}=S_{\mathrm{Lr}}^{2,43}
$$


Int. Jnl. of Multiphysics Volume 7 - Number $4 \cdot 2013$

351

$$
\begin{aligned}
& k_{r \mathrm{~L}}=S_{\mathrm{Lr}}^{2.92} \quad\left(\text { if } S_{\mathrm{Lr}} \geq 0.3\right) \\
& k_{r \mathrm{~W}}=\left[\frac{\left(S_{\mathrm{W}}-S_{W i}\right)}{\left(1-S_{W i}\right)}\right]^{4}
\end{aligned}
$$


